\title{
Sakpata, ein Beitrag afrikanischer Medizinmänner zur Pockeneradikation
}

\author{
Von Werner H.Stöcklin
}

Auf einer Reise durch Togo und Benin konnte ich vor einigen Jahren eine verschimmelte Statuette erwerben, die als Pockenfetisch oder «Sakpata» bezeichnet wurde (Abb. 1 und 2).

Fasziniert von der Häßlichkeit und den seltsamen Attributen dieser Skulptur, beschloß ich, ihrem Geheimnis etwas auf den Grund zu gehen. Der vorliegende Bericht stellt das Ergebnis meiner Nachforschungen dar, die sowohl exotische wie auch altvertraute Kapitel der Pockengeschichte berührten.

Die Experten, von denen hier die Rede sein soll, haben glücklicherweise ausgedient. 1977 konnten die Pocken von der WHO aus dem kosmopolitischen Krankheitsregister gestrichen werden.

Der letzte Patient, der an dieser Seuche zu leiden hatte, war Ali Maow Maalin aus Somalia ${ }^{1}$. Seit seiner Genesung wurden zwar noch zahlreiche Verdachtsfälle gemeldet, doch keiner davon konnte als echte Variola klassifiziert werden ${ }^{2}$.

\section{Europa}

Unter den Vorkämpfern unseres westlichen Medizinsystems war Rhazes (860-932) wohl der erste, der die Pocken als ein eigenständiges Syndrom erkannte und sie klinisch von den oft ähnlich verlaufenden Masern abzugrenzen versuchte ${ }^{3}$. Hinsichtlich ihrer Ursache hielt er die beiden Krankheiten allerdings für identisch, indem er sie mit der Theorie der «Säfteverderbnis durch das im Kind noch nicht genügend abgekühlte Blut» erklärte ${ }^{4}$.

Aus den kausalen Erwägungen von Rhazes ließen sich keine vorbeugenden Maßnahmen ableiten.

Erst Thomas Sydenham (1624-1689) vermochte etwas Licht in die epidemiologischen Aspekte der Pocken zu bringen. Einem neuen Konzept entsprechend, führte er sie als «akute Krankheit» auf einen «schädlichen von außen kommenden Stoff» zurück - im Gegensatz zu den chronischen 
Krankheiten, die, wie er aufgrund seiner eigenen Erfahrungen mit der Gicht annahm, aus einem von innen kommenden Stoff resultierten ${ }^{5,6}$.

Hermann Boerhaave (1668-1738) ging in seiner epidemiologischen Enträtselung der Pocken noch einen wesentlichen Schritt weiter ${ }^{7}$. Er hielt fest, daß dieses Leiden «durch Mittheilung eines contagiums von einem früher betroffenen Menschen erworben» werde, und präzisiert hierzu: «Das contagium scheint zuerst in der Luft enthalten zu sein und dann den Lungen, dem Mund, der Nase, der Speiseröhre, dem Magen und den Därmen mitgetheilt zu werden.. ${ }^{8}$

Zu einer eigentlichen Pockenprophylaxe vermochte allerdings auch diese miasmatische Theorie keinen Anlaß zu geben. Die Ansätze zur Eindämmung der Krankheit blieben in Europa äußerst bescheiden. Gelegentlich wurde die Isolierung von Patienten oder das Verlassen von Pockenhäusern als Schutzmaßnahme geübt. Und vereinzelt kannte man das Pockenkaufen, wie es etwa von Vollgrad 1671 beschrieben wird: «Man schickte die Kinder, welche die Pocken durchmachen sollten, in ein Blatternhaus, wo sie gegen Entgelt etwas Blatternschorf empfangen und in der Hand fest zusammendrücken konnten. ${ }^{9}{ }^{9} \mathrm{Im}$ übrigen aber fand man sich damit ab, daß von Pocken und Liebe niemand verschont bleiben konnte.

Eine eigentliche Impfung, die sogenannte Variolation, wurde um 1718 durch Lady Mary Wortley Montagu (die Frau des britischen Botschafters in Konstantinopel) nach Europa eingeführt. Die gräko-türkische Methode, die sie propagierte, war zwar schon 1714 durch Timoni und 1715 durch Kennedy beschrieben worden (die ihr Wissen ebenfalls in Byzanz bezogen hatten). Doch das Verdienst der Lady Mary war es, durch die erfolgreiche Impfung ihrer eigenen Kinder auch gleich das Vertrauen in diese Pockenprophylaxe in England importiert zu haben ${ }^{10}$.

Der Variolation, bei welcher der perkutan applizierte Impfstoff direkt aus Pusteln eines akut erkrankten Pockenpatienten entnommen wurde, hafteten allerdings noch gewisse Gefahren und Mängel an, die dann durch Edward Jenner (1749-1823) mit Hilfe der Kuhpockenimpfung, der Vakzination, umgangen werden konnten. Auch er nahm meist eine Übertragung von Mensch zu Mensch vor, doch stammte das Impfmaterial aus Pusteln, die lediglich durch Kontakt mit harmlosen Kuhpocken entstanden waren ${ }^{11}$.

Als Jenner 1798 nach langjährigen Vorversuchen mit seiner neuen Methode an die wissenschaftliche Öffentlichkeit trat, wurde er zunächst mit Spott und Skepsis für seine Mühen belohnt. Er blieb aber zuversichtlich und schrieb 1801 in seiner Publikation "On the origin of the vaccine inoculation": 
«Nun zeigt es sich mit einer über jeden Streit erhabenen Deutlichkeit, daß die Ausrottung der Pocken, der furchtbarsten Geißel des Menschengeschlechts, das Ergebnis der Impfung sein muß.» ${ }^{12,13}$

Während man in Europa bis ins 18. Jahrhundert hinein den Pockenepidemien rat- und tatlos ausgeliefert war, fand sich in andern Erdteilen eine völlig andere Situation.

Asien

In Indien soll die Variolation schon seit undenklichen Zeiten bekannt gewesen sein. Und im Werk «Sactaya Grantham» wird - laut Buschan ${ }^{14}$ sogar die Vakzination beschrieben. Der Text soll etwa so lauten: «Die Pocken, welche durch die Flüssigkeit von Kuheutern hervorgebracht werden, bedingen eine leichte Krankheit. Der Geimpfte wird niemals unter Blattern zu leiden haben.»

In China geht die Variolation zumindest bis 6. Jahrhundert nach Christus zurück. Getrocknete Eiterkrusten wurden zu Pulver zerrieben und noch nach jahrelanger Konservierung mit Hilfe eines Blasrohres oder eines Wattestäbchens in die Nasen der Kinder eingeführt ${ }^{15}$.

Afrika

Auch in afrikanischen Gebieten war die Pockenprophylaxe durch Variolation weit verbreitet. Genauere Belege sind vor allem aus Nordafrika ${ }^{16}$, Ghana ${ }^{17}$, Mali ${ }^{18}$, Togo ${ }^{19}$ und Tanzania ${ }^{20}$ zu finden. Doch war die Variolation auch bei kongolesischen und südafrikanischen Stämmen anzutreffen.

Es ist nicht möglich, die Pockenprophylaxe in Afrika bis zu ihren Wurzeln zurückzuverfolgen. Immerhin dürfte der amerikanische Prediger und Hexenjäger Cotton Mather den Beweis erbracht haben, daß die Variolation zumindest in Westafrika schon vor 1716 bekannt gewesen war. Denn in jenem Jahre hörte er durch seinen Negersklaven Onisemus eine genaue Schilderung der afrikanischen Impfmethode, die deutlich von der byzantinischen Variolation abwich ${ }^{21,22}$.

Im Zuge der Kolonialisierung wurde (wie Gally 1910 berichtet) die Variolation in den meisten afrikanischen Ländern verboten ${ }^{23}$. Da aber die Pocken überall ein latentes Problem waren und die großangelegten Vakzina- 
tionskampagnen der weißen Beamten und Missionare aus technischen Gründen oft versagten (so daß allen Bemühungen zum Trotz immer wieder verheerende Pockenepidemien auftraten), behielt die traditionelle Variolation ihre Bedeutung bei. Erst die Einführung des temperaturbeständigeren lyophilisierten Impfstoffes ließ die Variolation auch in Afrika allmählich obsolet werden ${ }^{24}$.

Die Inokulationstechnik ist in den afrikanischen Ländern erstaunlich vielfältig, wie eine Darstellung von Imperato aus dem Gebiet von Mali zeigt $^{25}$. Es fällt hier auf, daß im allgemeinen flüßiger, frischer Bläscheninhalt Verwendung fand. Doch war zumindest in Ghana, Nigeria, Togo und Benin auch die Verwendung von pulverisiertem, während längerer Zeit aufbewahrtem Borkenmaterial üblich.

Als Impfstelle wurde besonders häufig der Vorderarm verwendet, angeblich, damit man sich anhand der Narbe schon bei der Begrüßung sogleich über den epidemiologischen Status eines Fremden orientieren konnte.

Die Variolation wurde nicht von jedem beliebigen Stammesmitglied vorgenommen. In Mali waren alte Männer und Frauen, Schmiede und allenfalls die Lehrer der Koranschule für diese Tätigkeit ausersehen.

In Ghana, Togo und Benin war die Pockenprophylaxe und auch der Umgang mit Pockenpatienten einer speziellen Gruppe von Medizinmännern vorbehalten, die als Priester der Pockengottheit eingestuft wurden. Je nach dem sprachlichen Lokalkolorit hörte die Gottheit, welcher sie unterstanden (oder der zu dienen sie vorgaben), auf Namen wie Sakpata, Shopona, Chapanna oder Sabata. Bei den Yoruba war sie als Omolu oder Babalayé bekannt und gefürchtet ${ }^{27,28}$.

Die im Sakpata-Kult verwendeten Statuetten, die «Pockenfetische», sollen grundsätzlich alle der in Abb. 1 und 2 dargestellten Figur aus Benin geglichen haben. Durch einige Kaurimuscheln, die in ihrer symmetrischen Anordnung wie eine Verzierung erschienen, wurde der Hautausschlag symbolisiert. Einige mit Schnüren und Schlick am Bauch der Skulptur montierte Behälterchen aus Glas, Ton und Flaschenkürbis unterstrichen den spezifischen Aufgabenbereich dieses Fetischs, der primär dazu bestimmt war, gegen die Erkrankung an Blattern zu schützen.

Sofern die Angaben der Gewährsmänner in Togo stimmten, beinhaltete das mit einem Schraubdeckel versehene Fläschchen ursprünglich Kölnisches Wasser (das übrigens in Europa einst als wirksames Abwehrmittel gegen die Pest gepriesen wurde) ${ }^{29}$. In der Kürbisflasche - auf dem Rücken des Fetischs - befand sich wohlriechender Babypuder, der, auf die Haut 


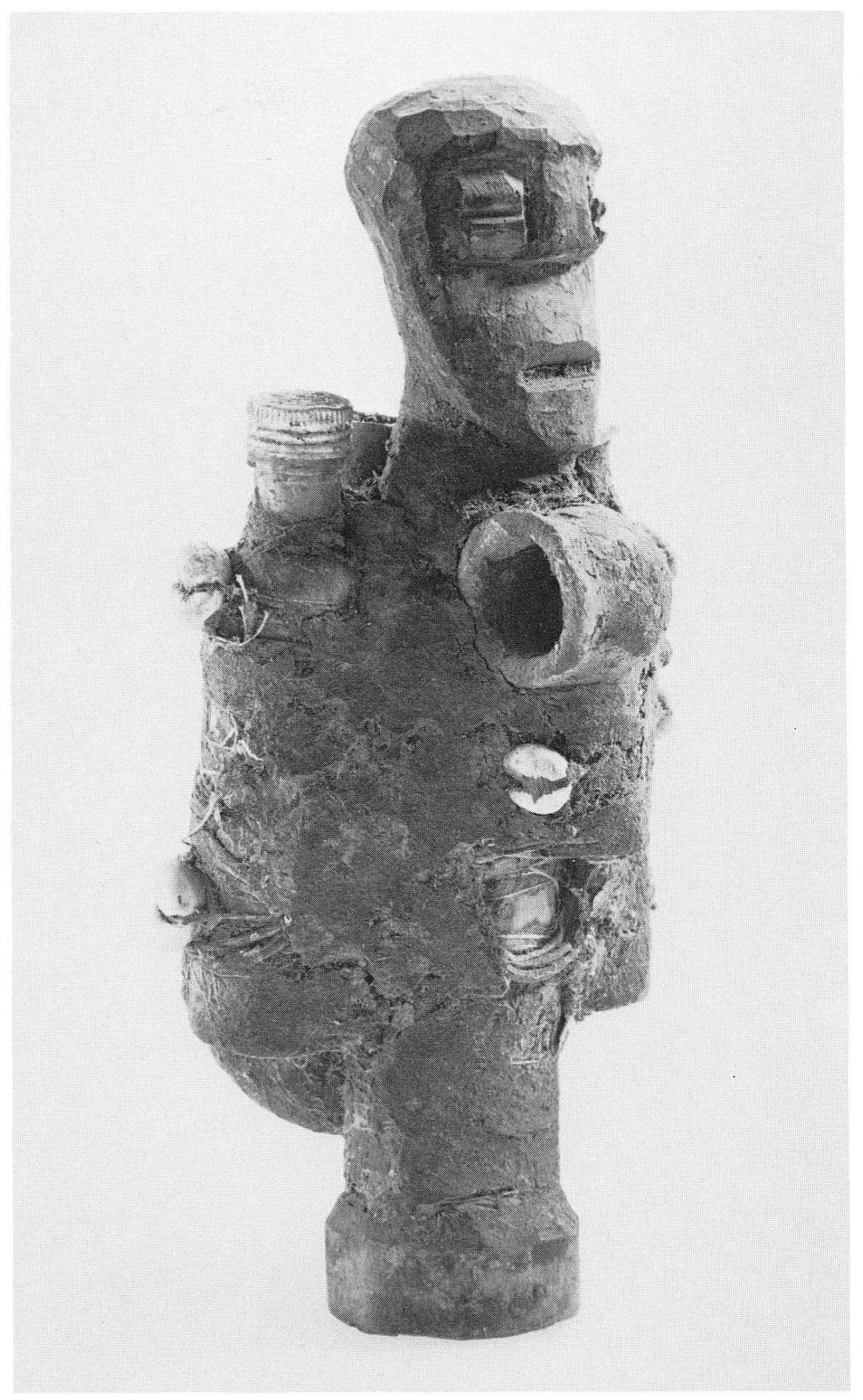

Abb.1. Pocken-Fetisch aus Benin 


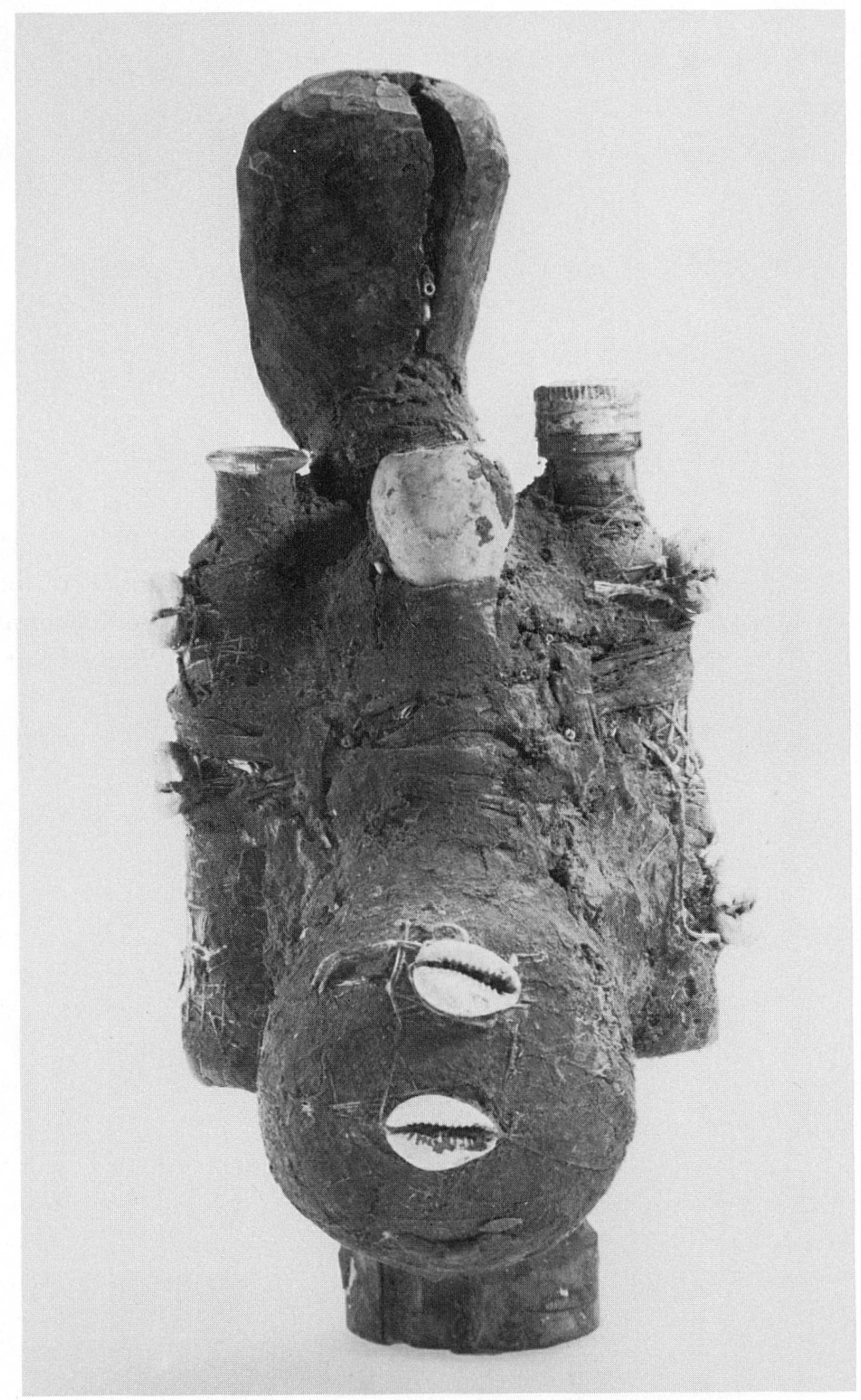

Abb. 2. Pocken-Fetisch aus Benin (dorsale Ansicht) 
gerieben, vor Auswirkungen von Hexerei und bösem Blick zu schützen vermochte. Im dritten, aus Ton geformten Gefäß wurde angeblich ein feiner Staub aus Pockenborken ${ }^{30}$ aufbewahrt, der nach Skarifizierung in die Haut des Impflings einmassiert wurde - wodurch eine Variolation zustande kam. Die Funktion des unverschlossenen Glasfläschchens (an der linken Seite der Statuette) konnte leider nicht eruiert werden.

Die Priester des Sakpata hatten ein relativ hohes soziales Ansehen. Ihre Aktionen waren von kultischem Geheimnis umhüllt. Der Umgang mit den Pocken - die bei einzelnen Stämmen als «Häuptling aller Krankheiten» bezeichnet wurden ${ }^{31}$ - gab ihnen eine große Macht, die sie sehr unberechenbar zu investieren pflegten.

Sie hatten das Monopol bei der Betreuung der Pockenkranken, konnten Hab und Gut der verstorbenen Pockenopfer einheimsen und nach Gutdünken die Kontaktpersonen impfen oder ungeimpft lassen. Glokpor behauptet sogar, daß die Sakpatapriester in Togo und Benin mit unsorgfältig durchgeführten Impfungen den Ausbruch neuer Epidemien provozieren konnten, wenn ihre Kasse nicht mehr stimmte, oder wenn der Rachedurst sie plagte $^{32,33}$.

Doch im positiven Sinne stand es immerhin auch in ihrer Willkür, eine ausgebrochene Epidemie durch Umgebungsprophylaxe einzudämmen und so die Pockenausrottung beschleunigen zu helfen.

Es mutet seltsam an, daß die Sakpata-Priester trotz ihrer fast sprichwörtlichen Skrupellosigkeit und Habgier generationenlang von der Gesellschaft geduldet oder gar geachtet wurden. Die Erklärung für dieses Phänomen ist wohl auf zwei Ebenen zu suchen. Einerseits liegt sie gewiß in der allgemeinen Furcht vor der Pockenkrankheit begründet. Anderseits aber darin, daß der Pockentod mancherorts in Afrika als «schlechter Tod» klassifiziert wurde. Auch Meningo-encephalitis, Hepatitis mit schwerem Ascites, Wochenbettfieber und maligne verlaufende Lepra endeten nach landläufiger Meinung oft mit dem «schlechten Tod». Es fällt nicht schwer zu vermuten, daß eine dumpfe Ahnung von Kontagiosität diesem Gliederungsprinzip zugrunde lag. Doch nach afrikanischem Krankheitsverständnis handelte es sich dabei um Leiden von Menschen, die durch Versündigung den Zorn unsichtbarer Mächte auf sich geladen hatten. Auch ein Patient, der sein Leben nicht in den Armen seiner Familienangehörigen beschließen konnte, war - wie Haaf von den Kusase berichtet - dem «schlechten Tod» geweiht. Damit war festgelegt, daß seiner Seele der Eintritt ins Totenreich oder ins Haus des Schöpfergottes versagt bleiben mußte. Die Hinterbliebenen 
versuchten den Tod des so schimpflich aus der Welt geschiedenen Stammesmitgliedes zu ignorieren. Sie beerdigten den Verstorbenen nicht in Ehren, wie dies sonst der Brauch war, sondern warfen ihn achtlos in ein altes, ausgedientes Grab oder gar in den Sumpf. Niemand richtete ein Totenfest und niemand begehrte die Habe des Toten. Die einzige Sorge der Lebenden war es, sich vor dem umherirrenden, auf Böses sinnenden Geist des Abgeschiedenen zu schützen, der nun natürlich auch andere mit in den Tod reißen wollte ${ }^{34}$.

Unter solchen Umständen schien es geradezu ein Segen für die Gesellschaft gewesen zu sein, daß sich wenigstens die Sakpata-Priester um die zu Recht gefürchteten Leichen der Pockenpatienten kümmerten und die Weiterverwendung ihrer materiellen Hinterlassenschaften übernahmen. Diese Gruppe von Medizinmännern konnte somit - zumindest bei einer guten Pflichtauffassung - nicht nur durch Variolationen die Pocken in Schach zu halten versuchen, sondern durch ihre Aneignung der kontagiösen Erbschaften und die Vermeidung von Totenfeiern Wesentliches zur Limitierung eines Seuchenherdes und damit letztlich zur Pockeneradikation beisteuern.

Selbst wenn - wie Glokpor ${ }^{35}$ und Fichte ${ }^{36}$ vermuten - die Habsucht die wichtigste Triebfeder bei der Tätigkeit gewisser Pockenpriester gewesen sein könnte, so müßte ihnen doch zugute gehalten werden, daß sie sich in rechter Gesellschaft befanden. Auch im vorbildlichen christlichen Europa scheinen ja die ökonomischen Aspekte bei der Betreuung der Pockenpatienten kräftig mitgespielt zu haben. In diesem Sinne möchte ich abschließend ein paar Zeilen aus einem Brief der Lady Montagu zitieren, den sie 1717 an ihre Freundin Miss Chiswell geschrieben hat: «Ich bin Patriotin genug, um mich zu bemühen, dieser heilsamen Entdeckung (der Variolation) in England Verbreitung zu verschaffen, und werde nicht verfehlen, einigen unserer Ärzte ganz ausführlich darüber zu schreiben, sobald ich irgendeinen unter ihnen kennenlerne, den ich für hinreichend selbstlos halte, einen ihrer beträchtlichsten Erwerbszweige zum Wohle der Menschheit zu vernichten. Doch diese Krankheit ist für sie zu einträglich, als daß sie nicht ihren ganzen Groll auf jenen verwegenen Wicht ausschütten sollten, der es unternähme, sie auszurotten .... ${ }^{37}$ 


\section{Anmerkungen}

${ }^{1}$ Donald A.Henderson, Smallpox-epitaph for a killer? Geogr. Magazine 154/6 (1978) S. 796-805.

${ }^{2}$ Dieter Stürchler, Epidemiegebiete tropischer Infektionskrankheiten, Bern (1981) S.38: Von Januar 1978 bis Januar 1980 wurden 144 Pocken-Verdachtsfälle gemeldet. Es handelte sich dabei um a) typische oder atypische Varizellen (z. B. der Pockenfall vom April 1980 aus Italien); b) generalisierte Vaccinia (Brit.med.J. 265-79, 1398/9; c) Affen-, Kamel- und Ziegenpocken beim Menschen; d) andere exanthematische Krankheiten.

${ }^{3}$ Rhazes, Über die Pocken und über die Masern. Klassiker der Medizin, Bd. 12, Leipzig (1911) S.11.

${ }^{4}$ Erwin Ackerknecht, Geschichte und Geographie der wichtigsten Krankheiten. Stuttgart (1963) S. 56.

5 Thomas Sydenham, Opera universa. Editio novissima Lugduni Batavorum (1674).

${ }^{6}$ P. Kübler, Geschichte der Pocken und der Impfung. Berlin (1901) S. 105.

${ }^{7}$ Hermann Boerhaave, Opera omnia medica. Venedig (1735).

${ }^{8}$ Zit. nach P. Kübler, l.c., S.111.

${ }^{9}$ P. Kübler, l.c., S. 115-6.

${ }^{10}$ E. Ackerknecht, l.c., S.58. Vgl. auch:

Genevieve Miller, Putting Lady Mary in her place: a discussion of historical causation. Bulletin of the History of Medicine 55 (1981) S.2-16.

${ }^{11}$ Edward Jenner, An inquiry into the causes and effects of the variolae vaccinae. London (1798).

12 Zit. nach P. Kübler, l.c., S. 165.

13 Jenners Optimismus gab offenbar auch den Anstoß zu folgendem Werk: A.F.Hecker, Die Pocken sind ausgerottet! Ein Handbuch für Ärzte und Nichtärzte, die die Geschichte der Kuhpocken in ihrem ganzen Umfange kennenlernen und die Impfung der Schutzblattern, die größte Entdeckung des achtzehnten Jahrhunderts, zweckmäßig anwenden und befördern wollen. Erfurt (1802).

${ }^{14}$ Georg Buschan, Über Medizinzauber und Heilkunst im Leben der Völker. Berlin (1941) S. 593 .

15 P. Kübler, l.c.S. 116.

${ }^{16}$ J.G. Schenchzer, An account of the success of inocculating the smallpox in Great Britain for the year 1727 and 1728. London (1729) S.61.

${ }^{17}$ D. Scott, Epidemic disease in Ghana. Oxford University Press, Oxford (1962) S. 120.

18 P. J. Imperato, Observations on variolation practices in Mali. Trop. geogr. Med., 26 (1974) S. 429-440.

${ }^{19}$ G.F. Glokpor, Seminar on smallpox eradication and measles control in Western and Central Africa. Proceedings of a meeting held in Lagos, Nigeria, May 13-20, 1969. U.S. Department od Health, Education and Welfare, Public Health Service, Center for Disease Control, Atlanta (1969) S. 59.

${ }^{20}$ C. W. Dixon, Smallpox. London (1951) S. 109.

${ }^{21}$ G. L. Kittredge, Some last works of Cotton Mather. Proc. Mass. Hist. Society, XLV (1911) S. 418 .

${ }^{22}$ E. Ackerknecht, l.c. S. 59 . 
${ }^{23}$ L. Gally, La lutte contre la variole en A. O. F. Larose, Paris (1910) S. 38

${ }^{24}$ P. J. Imperato, l.c., Tab. IV, S. 432.

${ }^{25}$ P. J. Imperato, l.c., Tab.III, S.433!

${ }^{26}$ G. F. Glokpor, l.c.

${ }^{27}$ E. Haaf und J.Zwernemann, Geburt - Krankheit - Tod in der afrikanischen Kunst. Stuttgart/New Xork (1975) S.26.

${ }^{28}$ Hubert Fichte und M. R. Amakoue, Ethnomedizin, Ethnobotanik und Ethnopharmakologie in Togo, ein Gespräch. Ethnomed. V, 1/2 (1978/79) S. 163/4.

${ }^{29}$ E. Ackerknecht, l.c. S. 11.

${ }^{30}$ Offenbar handelt es sich bei diesem «Staub» um Überreste einer «Impfsalbe», deren Herstellung von Haaf (l.c., S.28) wie folgt beschrieben wird: «Nach eigenen Informationen, die wir von einer hochgestellten initiierten Persönlichkeit in Abomey (Dahomey) erhielten, wird das Pockensekret Erkrankter mit (Medizin) vermischt und dem Impfling nach vorheriger Skarifikation in die Haut eingerieben. Die «Medizins soll aus Rindergalle, Urin eines Ziegenbocks und zwei Pflanzen bestehen.»

${ }^{31}$ Ernst Haaf, Die Kusase, eine medizinisch-ethnologische Studie über einen Stamm in Nordghana. Gießener Beiträge zur Entwicklungsforschung, Stuttgart (1967) S. 154.

${ }^{32}$ G.F. Glokpor, l.c.

${ }^{33}$ P. J. Imperato, l.c. S. 438.

${ }^{34}$ E. Haaf, l.c. (1967) S.99/100 und 154.

${ }^{35}$ G.F. Glokpor, 1.c.

${ }^{36}$ H. Fichte und M. R. Amakoue, l.c., S. 163.

${ }^{37}$ Zit. nach P. Kübler, l.c., S.120/1.

\section{Zusammenfassung}

Während in Europa erst im Verlauf des 18. Jahrhunderts wirksame Schutzmaßnahmen gegen die Pocken eingeführt wurden (Variolation 1718, Vakzination 1798), reicht die Geschichte der Pockenprophylaxe in China, Indien und Afrika sehr viel weiter zurück.

In Westafrika wurde das Auftreten der gefürchteten Pockenseuche meist mit einer mächtigen Gottheit (Sakpata, Chapanna, Omolu oder Babalayé genannt) in Zusammenhang gebracht.

Dem Kult dieser Gottheit diente eine spezielle Priesterschaft. Eine wichtige Vermittlerrolle zwischen Mensch, Gott und Krankheit wurde dem Pockenfetisch zugedacht, einer hölzernen Skulptur, die als bedeutsame Attribute kleine Gefäße (Kalebassen, Flaschen, Affenschädel) auf sich trug. Diese Behälterchen waren u.a. zur Aufnahme von Pockenschorf bestimmt, welcher - mit Kräutern und Essenzen vermischt - als Impfmaterial Verwendung fand (Variolation).

Die Pockenpriester hatten das «Monopol» bei der Betreuung der Pockenpatienten inne und konnten den gesamten Besitz der verstorbenen Klienten für sich beanspruchen.

Die ambivalente Einstellung der Bevölkerung dieser als raffsüchtig und eigenmächtig bekannten Priesterschaft gegenüber wird unter dem Aspekt des «schlechten Todes» näher erläutert. 


\section{Summary}

Much earlier than in Europe effective protection against smallpox was known in China, India and Africa. In this article traditional variolation in Westafrica is being discussed with special reference to the socalled priests of the smallpox-goddess (Sakpata, Chapanna, Omolu, Babalayé).

Dr. med. Werner H. Stöcklin

Rößligasse 32

CH-4125 Riehen 